\title{
Properties of TaN Films for ULSIs Prepared by Reactive Sputter Deposition
}

\author{
H. Tajima*, N. Shiobara, H. Katsumata and S. Uekusa \\ Department of Electronics and Bioinformatics, School of Science and Technology, Meiji University, \\ 1-1-1 Higashimita, Tama-ku, Kawasaki, Kanagawa 214-0033, Japan. \\ uekusa@isc.meiji.ac.jp
}

(Received: October 7, 2010 ; Accepted: February 17, 2011)

\begin{abstract}
Tantalum Nitride (TaN) thin films were deposited onto n-type $\mathrm{Si}(100)$ and (111) substrates with $\mathrm{SiO}_{2}$ films at room temperature by radio frequency (RF) magnetron sputtering under $\mathrm{Ar}-\mathrm{N}_{2}$ plasma using a tantalum target. We observed the formation of TaN thin films with a wide range of the electrical resistivity as a function of the $\mathrm{N}_{2}$ gas flow ratio, working pressure and the sputtering power, and their origins are discussed based upon the structural properties and chemical compositions of TaN thin films before $\mathrm{Cu}$ films deposition. In this study, we first observed that the TaN thin films with lower resistivity exhibited $\mathrm{TaN}(200)$ preferentially oriented structures and had larger grain sizes. It was also found that the formation of $\mathrm{TaO}(002)$ made the resistivity of TaN thin films higher because of the diffusion of oxygen from $\mathrm{SiO}_{2}$ films during deposition.
\end{abstract}

\section{Introduction}

Tantalum nitride (TaN) has many attractive characteristics, such as good chemical stability, high hardness, high melting point, and low electrical resistivity and high thermal conductivity, which make it possible to use as diffusion barriers for wiring techniques in silicon-based integrated circuits such as ULSI [1-4]. Moreover, TaN can be used in wide variety of applications such as corrosion-resistant materials and high-speed thermal printing head as well as thin film resistors $[2,3]$. We have studied reactive sputter deposition and properties of $\mathrm{TaN}$ thin films on $\mathrm{Si}$ substrates $[2,3]$. In this study, we investigated the influence of the resistivity of TaN thin films deposited by RF magnetron sputtering on the $\mathrm{N}_{2}$ gas flow ratio, working pressure and sputtering power dependent, and the results were compared with their crystalline structures, electrical resistivity and chemical compositions.

\section{Experimental}

Tantalum nitride (TaN) thin films were deposited onto (100) and (111)-oriented n-type Si substrates with/without $\mathrm{SiO}_{2}$ films at room temperature by radio frequency (RF) sputtering under $\mathrm{Ar}-\mathrm{N}_{2}$ (both of Purity are more than $99.999 \%$ ) plasma using a tantalum (Ta) target (Purity of $99.99 \%, \phi$ 4inches, Kojyundo Chemical Laboratory Co., LTD), where the $\mathrm{SiO}_{2}$ films with a thickness of $0.5 \mu \mathrm{m}$ were thermally grown on Si substrates before depositions of TaN. The distance between the target and the substrate in sputtering chamber was about $42 \mathrm{~mm}$. Prior to TaN deposition, the target was cleaned by pre-sputtering in Ar plasma for 10min, while the sputtering time was kept constant for $30 \mathrm{~min}$. The thickness of deposited TaN thin films was in the range of $0.2 \sim 1.5 \mu \mathrm{m}$. The chamber was evacuated to the base pressure of $8.0 \times 10^{-5} \mathrm{~Pa}$ for each run. We investigated the effect of following three process parameters on film properties, i.e. $\mathrm{N}_{2}$ gas flow ratio, working pressure and sputtering power. Firstly, $\mathrm{N}_{2}$ gas flow ratio defined as $\left(\mathrm{N}_{2} / \mathrm{Ar}+\mathrm{N}_{2}\right) \times 100[\%]$ was varied in the range of $10 \sim 35 \%$, in which working pressure and sputtering power were maintained at $2.7 \mathrm{~Pa}$ and $200 \mathrm{~W}$ respectively. Secondly, working pressure determined by $\left(\mathrm{Ar}+\mathrm{N}_{2}\right)$ of total gas flow was changed for values ranging from $2.7 \sim 13.3 \mathrm{~Pa}$, while $\mathrm{N}_{2}$ gas flow ratio and sputtering power were kept constant at $20 \%$ and $200 \mathrm{~W}$ respectively. Thirdly, the sputtering power was varied from 150 to $300 \mathrm{~W}$ by keeping working pressure at $2.7 \mathrm{~Pa}$ and the $\mathrm{N}_{2}$ gas flow ratio at $20 \%$. Finally, the dependence of the type of $\mathrm{Si}$ substrates with/without $\mathrm{SiO}_{2}$ films was investigated at $\mathrm{N}_{2}$ gas flow ratio of $20 \%$, where the working pressure was 2.7 $\mathrm{Pa}$ and the sputtering power was chosen to be 
$200 \mathrm{~W}$. The structural properties were investigated through X-ray diffraction (XRD) using a $\theta-2 \theta$ method (Rigaku Co.) with a Cuk $\alpha$ radiation $(0.15406 \mathrm{~nm})$. The film thickness was measured by stylus surface profiler (Dektak 6M, ULVAC), and the electrical resistivity was measured at a room temperature by four-point probe method with a hall effect system (HL5500PC, ACCENT). The microstructure of thin films was observed using scanning electron microscopy with the power of 5 kV (SEM, Hitachi S5200 or Hitachi SU6600) and the chemical composition of cross-sectional part of TaN thin films was analyzed using energy dispersive X-ray spectrometry (EDX, Thermo electron, Noran System SIX NSS 102).

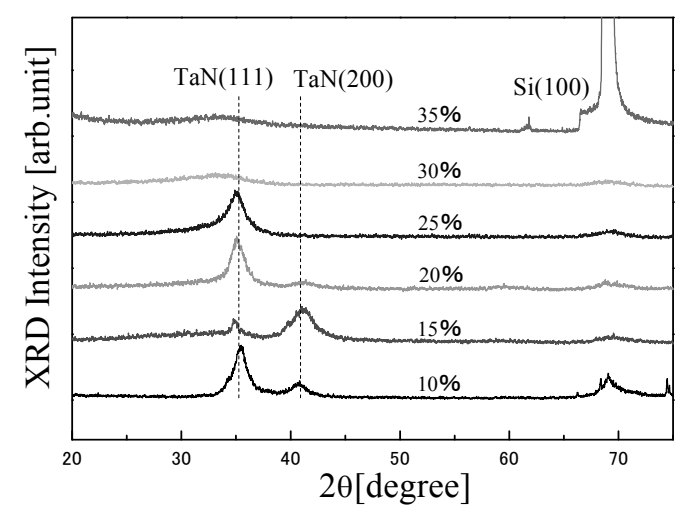

Fig .1. The dependence of $\mathrm{N}_{2}$ gas flow ratio on XRD spectra for TaN thin films on $\mathrm{SiO}_{2} / \mathrm{Si}(100)$.

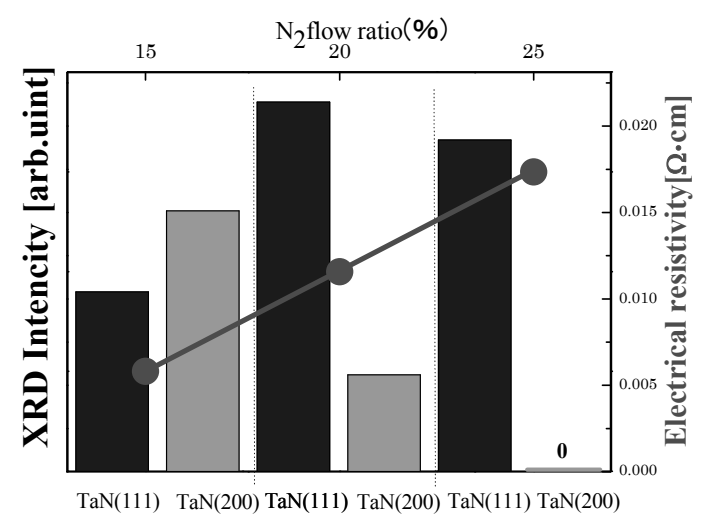

Fig. 2. The XRD intensity and electrical resistivity vs. $\mathrm{N}_{2}$ gas flow ratio for $\mathrm{TaN}$ films on $\mathrm{SiO}_{2} / \mathrm{Si}(100)$.

\section{Results and discussion}

\subsection{Influence of the $\mathrm{N}_{2}$ gas flow ratio}

Figure 1 shows the dependence of $\mathrm{N}_{2}$ gas flow ratio on XRD spectra for TaN thin films deposited on $\mathrm{SiO}_{2} / \mathrm{Si}$ (100). In Fig. 1, the two main peaks observed at 35.9 degrees and 41.6 degrees originate from the cubic structures with $\mathrm{TaN}(111)$ and $\mathrm{TaN}(200)$ orientations. Figure 2 shows the dependencies of $\mathrm{N}_{2}$ gas flow ratio on the XRD intensity and the resistivity for TaN thin films deposited on $\mathrm{SiO}_{2} / \mathrm{Si}(100)$. Over $\mathrm{N}_{2}$ gas flow ratio of $15 \%$, resistivity was increased with a decrease in $\mathrm{TaN}(200)$ intensity and increase in $\mathrm{TaN}(111)$ intensity as shown in Fig.2. From these results, we can conclude that the appearance of $\mathrm{TaN}(200)$ peak makes resistivity lower than that of $\mathrm{TaN}(111)$.

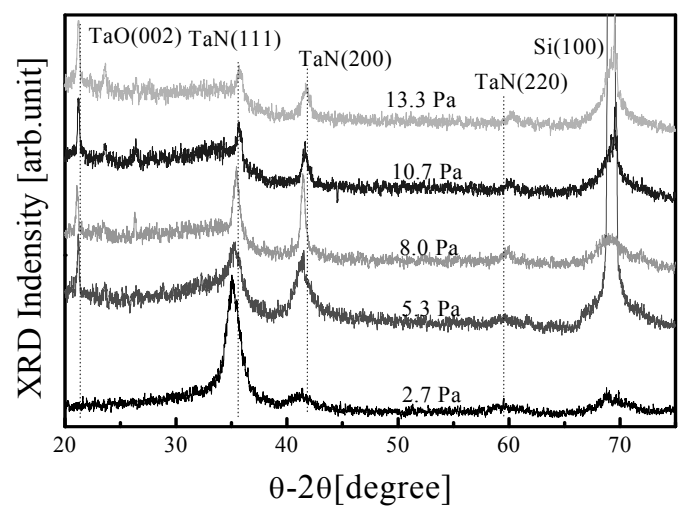

Fig. 3. The dependence of working pressure on XRD spectra for TaN thin films on $\mathrm{SiO}_{2} / \mathrm{Si}(100)$.

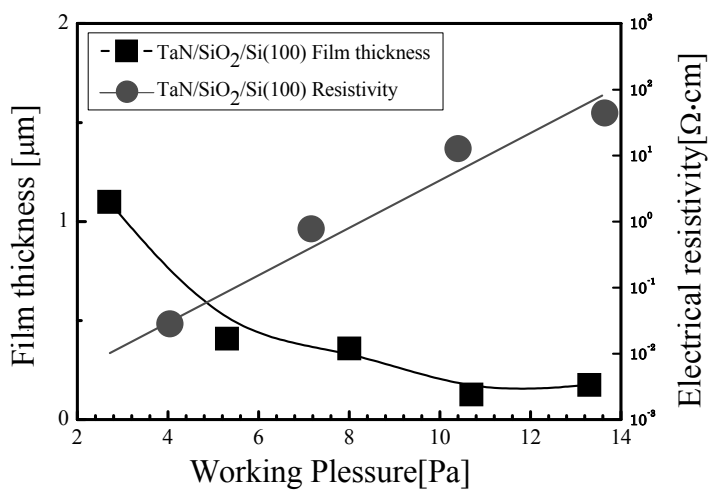

Fig. 4. The film thickness and the electrical resistivity vs. the working pressure for $\mathrm{TaN}$ films on $\mathrm{SiO}_{2} / \mathrm{Si}(100)$.

\subsection{Influence of working pressure}

XRD spectra for TaN thin films deposited on $\mathrm{SiO}_{2} / \mathrm{Si}(100)$ are shown in Fig.3 as a function of working pressure. From Fig. 3, one can see that the peak intensities of $\mathrm{TaN}(111)$ and $\mathrm{TaN}(200)$ decrease and the peak intensity of rhombic structure of $\mathrm{TaO}(002)$ at 21.3 degrees increases with an increase in working pressure. With all range of working pressure, the peak intensity of cubic structures of $\mathrm{TaN}(220)$ at 60.3 degrees was almost same. Figure 4 shows the film thickness and the electrical resistivity for $\mathrm{TaN}$ thin films on $\mathrm{SiO}_{2} / \mathrm{Si}(100)$ as a function of working pressure. It is found from Fig.4 that with increasing working pressure, the film thicknesses decreased slightly, whereas the electrical resistivity was increased 
exponentially. In order to clarify the reason of an increase exponentially, $\mathrm{TaO}(002)$ peak intensity shown in Fig. 3 was normalized by TaN(200) peak intensity and the results are shown in Fig. 5. In Figs. 5 , the ratio of $\mathrm{TaO}(002)$ peak intensity gradually increased with increasing working pressure. Furthermore, it can be seen from Fig.3 that the intensity of $\mathrm{TaO}(002)$ becomes dominant with

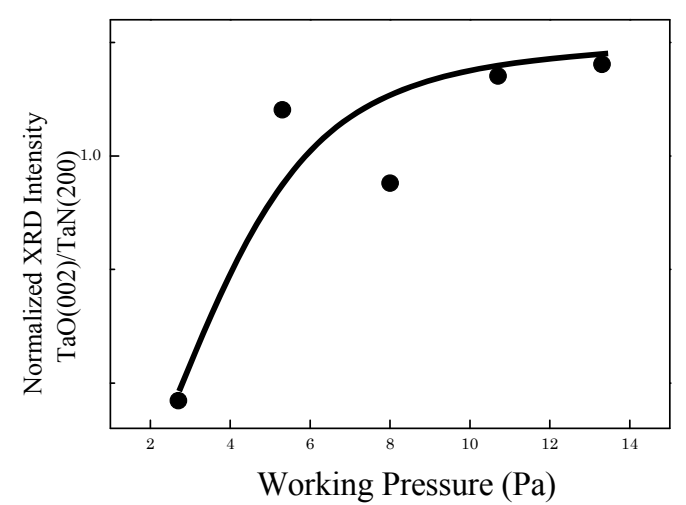

Fig. 5. Normalized XRD intensity, $\mathrm{TaO}(002) / \mathrm{TaN}(200)$ for TaN films on $\mathrm{SiO}_{2} / \mathrm{Si}(100)$ as a function of working pressure.

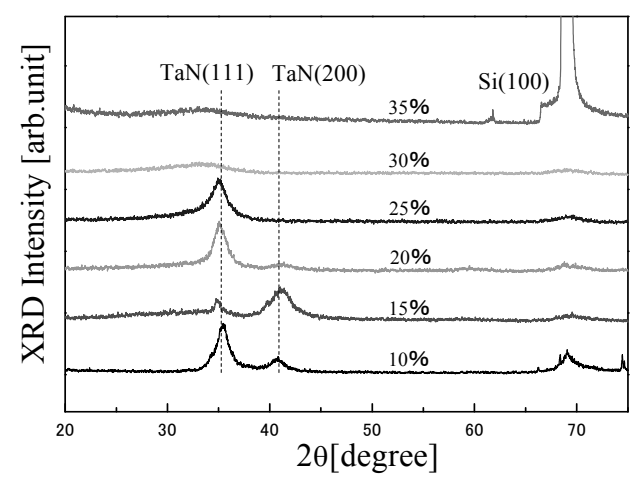

Fig. 6. The dependence of the sputtering power on XRD spectra for TaN thin films on $\mathrm{SiO}_{2} / \mathrm{Si}(100)$.

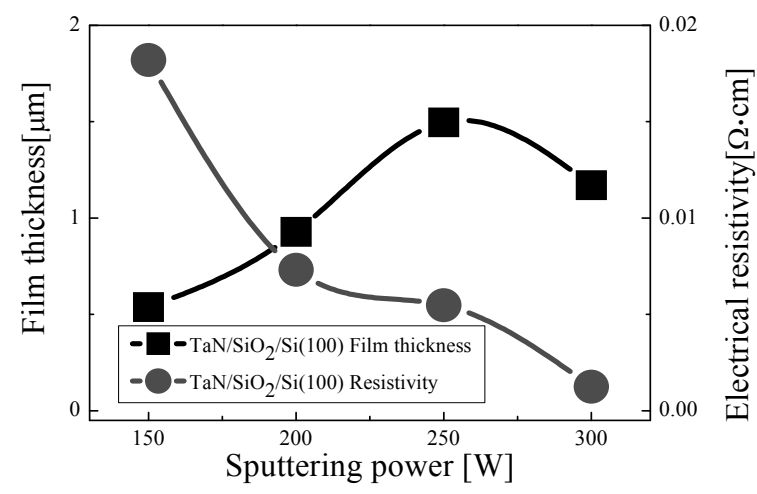

Fig. 7. The film thickness and the electrical resistivity vs. the sputtering power for $\mathrm{TaN}$ films on $\mathrm{SiO}_{2} / \mathrm{Si}(100)$.

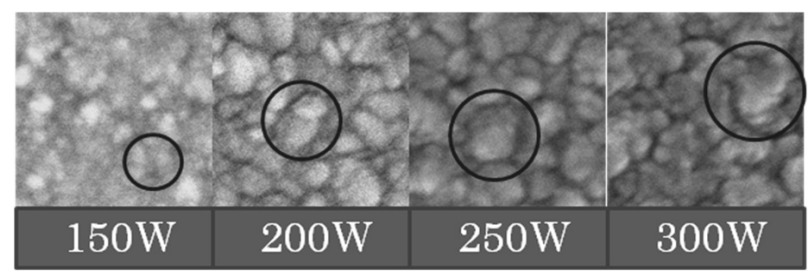

Fig. 8. The SEM surface morphologies of TaN thin films deposited on $\mathrm{SiO} 2 / \mathrm{Si}(100)$ with different sputtering powers.

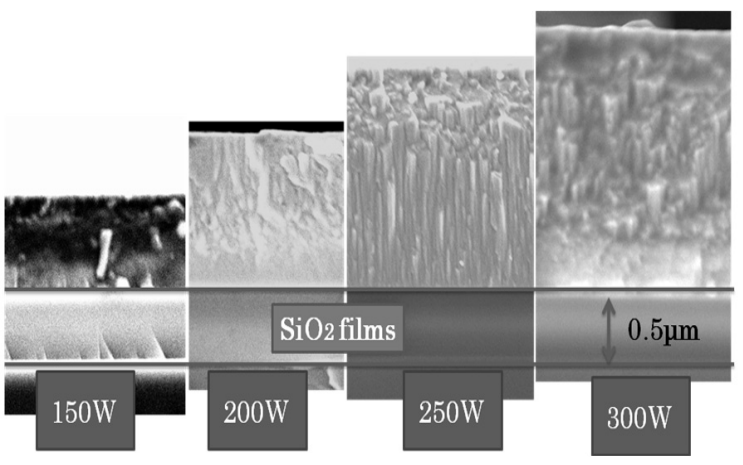

Fig. 9. The cross-sectional SEM images of TaN thin films deposited on $\mathrm{SiO} 2 / \mathrm{Si}(100)$ with different sputtering powers.

increasing working pressure. We assume the creation of $\mathrm{TaO}(002)$ were made from the diffusion of oxygen from $\mathrm{SiO}_{2}$ films as discussed in sec 4 . Thus, we conclude the origin of great increase in resistivity is due to a creation of tantalum oxide with $\mathrm{TaO}(002)$ orientation.

\subsection{Influence of the sputtering power}

Figures 6 and 7 show the dependences of the sputtering power on XRD spectra and film thickness and electrical resistivity for $\mathrm{TaN}$ thin films on $\mathrm{SiO}_{2} / \mathrm{Si}(100)$, respectively. With increasing sputtering power, intensity of $\mathrm{TaN}(111)$ peak became weaker while that of $\mathrm{TaN}(200)$ peak became stronger. As for the resistivity of TaN thin films, it decreased with increasing sputtering power. These results are consistent with that the appearance of $\mathrm{TaN}(200)$ peak makes resistivity lower than that of $\mathrm{TaN}(111)$ as discussed in sec 1. The intensity of $\mathrm{TaN}(220)$ was almost same with all range of sputtering power. In Fig.7, the film thicknesses were found to be thicker as the sputtering power increased, but the films peeling off were seen at $300 \mathrm{~W}$ and consequently we got the unexpected measurement values of film thickness and electrical resistivity as shown in Fig.7. Figure 8 and 9 show the SEM surface morphologies and the cross-sectional SEM images of the TaN thin films deposited on $\mathrm{SiO}_{2} / \mathrm{Si}(100)$. In Fig. 8, the grain sizes were observed to be bigger with increasing the sputtering power. The average grain sizes at 150 , 
200,250 and $300 \mathrm{~W}$ were estimated to be $52 \mathrm{~nm}$, $86 \mathrm{~nm}, 184 \mathrm{~nm}$ and 292nm, respectively. In Fig.9, columnar structures were clearly observed above $250 \mathrm{~W}$ and their diameters increased with increasing with sputtering power. It is thought that the amount of $\mathrm{Ta}$ atoms with high kinetic energies increases due to an increase in sputtering yield and the reaction of Ta atoms with nitrogen becomes active,

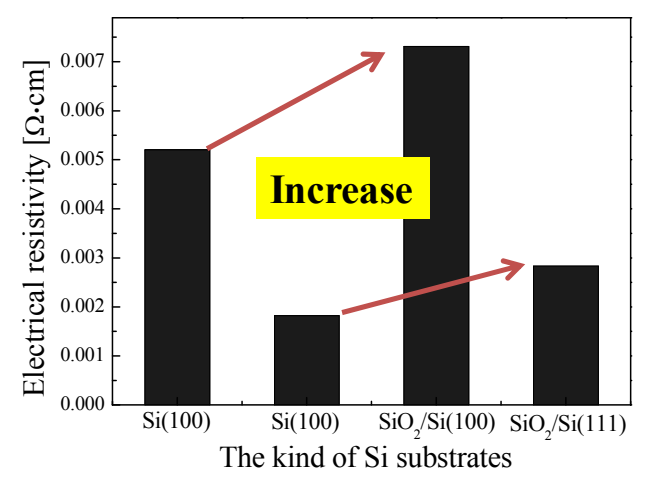

Fig.10. the electrical resistivity for TaN films with different types of substrates.

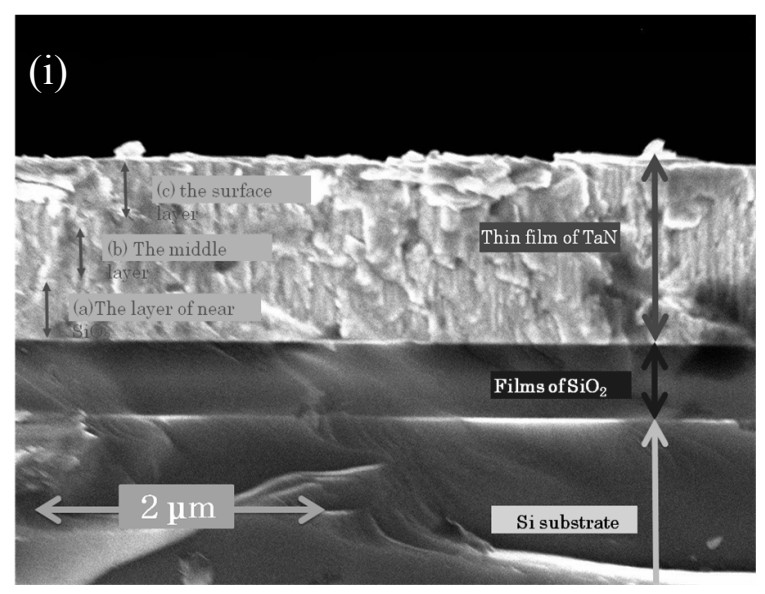

(ii)

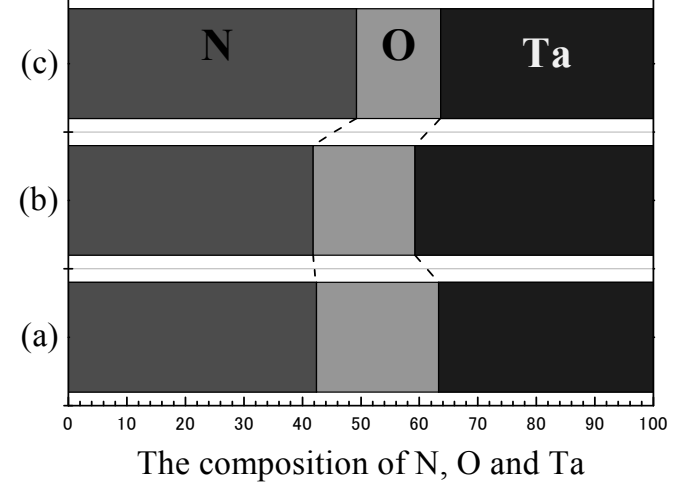

Fig. 11. The cross-sectional SEM image (i) and the composition of N,O and Ta measured by EDX (ii) for TaN films deposited on $\mathrm{SiO} 2 / \mathrm{Si}(100)$ substrates, which can be divided into three stages; (a), (b) and (c). which stimulates surface migration. Through this process, the $\mathrm{TaN}$ columnar sizes are larger with increasing sputtering power, which makes the resistivity lower as was shown in Fig.7.

\subsection{The dependence of the substrates with or without $\mathrm{SiO}_{2}$ layer}

Figure 10 shows the electrical resistivity for TaN thin films deposited on the $\mathrm{Si}(100), \mathrm{Si}(111)$, $\mathrm{SiO}_{2} / \mathrm{Si}(100)$, and $\mathrm{SiO}_{2} / \mathrm{Si}(111)$. All of film thicknesses were almost same, however, the resistivity were remarkably increased for the TaN films with $\mathrm{SiO}_{2}$ layers in comparison with those without $\mathrm{SiO}_{2}$ layers. Figure 11 shows the cross-sectional SEM image (i) and the composition ratio measured by EDX (ii), for TaN films deposited on $\mathrm{SiO}_{2} / \mathrm{Si}(100)$ substrates which were divided into the three stages; (a) the layers near the $\mathrm{SiO}_{2}$ layers, (b) the middle layers and (c) the surface layers. From these measurements, it was found that the composition ratios of oxygen were increased in regions which are closer to $\mathrm{SiO}_{2}$ layers. We conclude that the diffusion of oxygen from $\mathrm{SiO}_{2}$ layer into TaN films is the reason of an increase in resistivity for TaN films deposited on $\mathrm{SiO}_{2}$ as shown in Fig.12.

Figure 12 shows the cross-sectional SEM image of $\mathrm{TaN} / \mathrm{SiO}_{2} / \mathrm{Si}$ and the EDX mapping of $\mathrm{O}, \mathrm{N}$ and Ta. Fig. 11(ii) indicated the diffusion of oxygen from $\mathrm{SiO}_{2}$ layer into TaN thin films, whereas in Fig.12, the mapping of nitrogen shows nitrogen shortage in $\mathrm{TaN}$ thin films near the $\mathrm{SiO}_{2}$ layers. From these EDX mapping, we suppose that TaN thin films near the $\mathrm{SiO}_{2}$ layers include the $\mathrm{TaO}(002)$ oriented layers, which was evidenced by XRD. This result could be convincing because tantalum is known to be an element which can be relatively easier to oxidize than silicon. We guess that the sputtered Ta atoms come to $\mathrm{SiO}_{2}$ surface and then oxygen atoms diffused from $\mathrm{SiO}_{2}$ layer are subjected to a chemical reaction with $\mathrm{Ta}$ atoms, which eventually create tantalum oxides with $\mathrm{TaO}(002)$ orientation.

\section{Conclusion}

We investigated the electrical resistivity, the crystallographic structure, the film thickness, and the surface morphology of TaN thin films deposited by reactive RF sputtering. It was found that the resistivity, film thickness, and crystallographic structure of TaN thin films could be controlled by changing $\mathrm{N}_{2}$ gas flow ratio, working pressure and sputtering power for the application to ULSI. In $\mathrm{N}_{2}$ gas flow ratio of $15 \sim 25 \%$, electrical resistivity of $\mathrm{TaN}(200)$ was lower than that of $\mathrm{TaN}(111)$. With 
increasing sputtering power, the resistivity decreased with increasing grain size and the peak intensity of $\mathrm{TaN}(200)$. With increasing working pressure, the electrical resistivity increased due to formation of $\mathrm{TaO}(002)$. When $\mathrm{TaN}$ was deposited on $\mathrm{SiO}_{2}$ films, the diffusion of oxygen from $\mathrm{SiO}_{2}$ layer into $\mathrm{TaN}$ was found and consequently the resistivity increased.

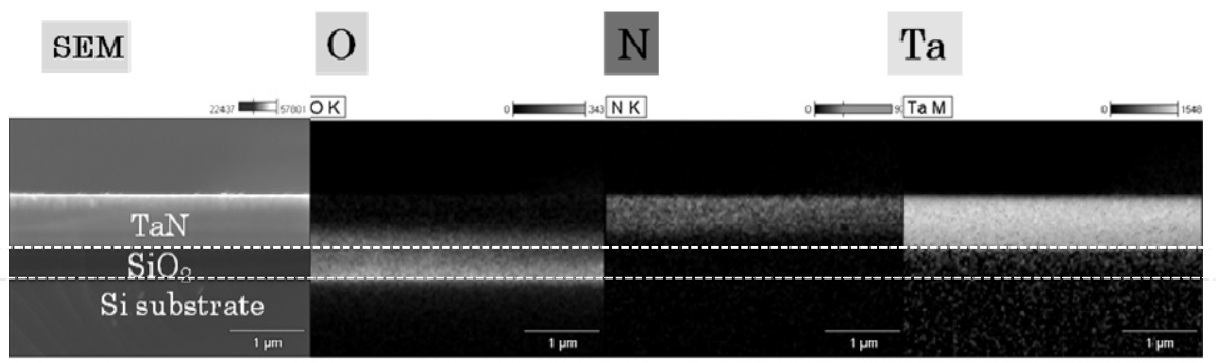

Fig. 12. The cross-section SEM image, the EDX mappings of $\mathrm{O}$, Ta and $\mathrm{N}$ for TaN thin films deposited on $\mathrm{SiO}_{2} / \mathrm{Si}$ substrates.

\section{Acknowledgements}

We are grateful for the personnel of Hitachi High-Technologies Corporation, who provided us the EDX mapping data.

\section{References}

[1] S.M. Kang, S.G. Yoon, S.J. Suh and D.H. Yoon, J. Thin Solid Films, 6 (2006) 113.
[2] S. Uekusa and T. Hama, Mem. Inst. Sci. Tech. Meiji Univ. 46(2) (2007) 19.

[3] T. Hama, S. Uekusa, 4th Vacuum and Surface Sciences Conference of Asia. and Australia Program \& Abstracts (2008) 382.

[4] N. Frety, J. Nazon, J. Sarradin, V. Flaud, J.C. Tedenac, J. Alloys and Compounds, 464 (2008) 526. 